\title{
Efficient Implementation of Multistage Digital Interpolators Using Half Band Filters
}

\author{
R. Ratan*, S. Sharma And A.K. Kohli \\ Department of Electronics \& Communication Engineering, Thapar University, Patiala, Punjab, 147004, India

\begin{abstract}
The process of increasing the sampling rate of any signal by a factor is known as interpolation. This paper presents the effective and efficient implementation of multistage implementation of digital interpolator using half band filters. The single stage filters are efficient for lower order interpolation factors but for higher rate change which is required in modern digital communication systems like wideband code division multiple access, worldwide interoperability for microwave access single stage implementation does not gives efficient response. For these applications a multistage implementation of the interpolator is preferred. But multistage implementation increases complexity of the overall system. This complexity can be reduced considerably by using half band filters. All this has been done with the help of the Agilent's advanced design software.
\end{abstract}

DOI: $10.12693 /$ APhysPolA.123.199

PACS: 84.30.Qi, 84.30.Vn

\section{Introduction}

In modern digital communication systems, the sampling rate conversion is an important process with the help of which, we can either decrease or increase the sampling rate. The process of decreasing the sampling rate is known as decimation and the process of increasing the sampling rate is known as interpolation.

\section{Interpolation (sampling rate up-conversion) by an integer factor $L$}

If the sampling rate is increased by an integer factor $L$, then the new sampling period $T^{\prime}$ may be expressed as in Eq. (1):

$$
\frac{T^{\prime}}{T}=\frac{1}{L},
$$

and the new sampling rate $F^{\prime}=L F$. This process of increasing the sampling rate of a signal $x(n)$ by $L$ implies that we must interpolate $(L-1)$ new sample values between each pair of sample values of $x(n)[1,2]$. Figure 1 shows the conceptual block diagram of single stage interpolator and Fig. 2 shows the block diagram of a multistage interpolator.

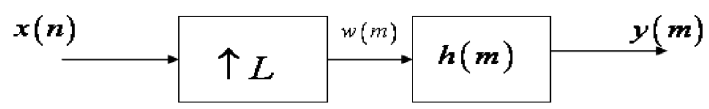

Fig. 1. Block diagram of interpolator.

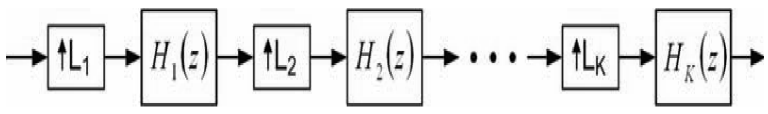

Fig. 2. Block diagram of multistage interpolator.

*corresponding author; e-mail: rajeevratan@aol.in

\section{Nyquist half band filters}

Digital $L$ th-band FIR filters are the special classes of digital filters, which are of particular interest both in single-rate and multirate signal processing [3]. The common characteristic of $L$ th-band low-pass filters is that the cutoff angular frequency is located at $\pi / L$, and the transition band is approximately symmetric around this frequency. In time domain, the impulse response of an $L$ th-band digital filter has zero valued samples at the multiples of $L$ samples counted away from the central sample to the right and left directions. Actually, an Lth-band filter has the zero crossings at the regular distance of $L$ samples thus satisfying the so-called zero intersymbol interference property. Sometimes the Lth band filters are called the Nyquist filters $[4,5]$. The important benefit in applying Lth band FIR filters is the efficient implementation, when every second coefficient in the transfer function is zero valued. Due to the zero intersymbol interference property, the Lth-band filters are very important for digital communication transmission systems. They are very popular in the sampling rate alteration systems as well, where they are used as interpolation filters in single-stage and multistage systems [6].

When the interpolation-by- $L$ is performed with an $L$ th-band filter, the original values of the input samples appear at the output without any distortion at the regular time intervals of $L$ samples [7]. The in between $L-1$ samples are determined by interpolation. It is well known that the interpolation by $L$ consists of two operations: up-sampling by $L$, and the low-pass filtering [8]. Hence, the samples $y[m]$ appearing at the interpolator's output are to be computed by convolving the up-sampled signal $\left\{x_{u}[m]\right\}$ with the low-pass filter impulse response $\{h[n]\}$, which may be shown as in Eq. (2)

$$
y[m]=L \sum_{k=0}^{2 k} h[k] x_{u}[m-k] .
$$

The sequence $\left\{x_{u}[m]\right\}$ contains $L-1$ zero-valued samples 
between each two nonzero samples, and the nonzero samples in $\left\{x_{u}[m]\right\}$ are those of the original signal $\left\{x_{u}[n]\right\}$.

A half-band filter is an $L$ th-band filter with $L=2$, and consequently, the half-band filter divides the base-band of the signal into two equal subbands. An equiripple half-band filter can be designed by using the ParksMcClellan-Rabiner algorithm, but for a large $\mathrm{N}$ this numerical procedure produces an error in the values of coefficients. A convenient method, named half-band filter trick, is described in Ref. [2], and in Ref. [1]. This method decreases the computational requirements by a factor of two. In the first step, a modified filter of about half the required length is designed using the Parks-McClellanRabiner program. In the second step, inserting zeros for the odd-index coefficients, scaling the even-index coefficients by 0.5 , and adding the central coefficient of 0.5 , creates the desired half-band filter. In this way, sufficient coefficient accuracy is achieved, and the design time is considerably decreased. An important advantage of a linear-phase half-band filter is the efficient implementation, which follows from two favorable properties of the filter impulse response:

- The number of nonzero-valued coefficients is nearly half of the filter length.

- The nonzero coefficients exhibit symmetry property.

\section{Experimental results and discussion}

Here we have connected three interpolators in cascade which consist of pulse shaping circuits (interpolated by 2) followed by two half band filters (each half band filter interpolated by 2) with the following specifications: sampling frequency $=46.08 \mathrm{MHz}$; bandwidth $=5 \mathrm{MHz}$; interpolation factor $=8$.

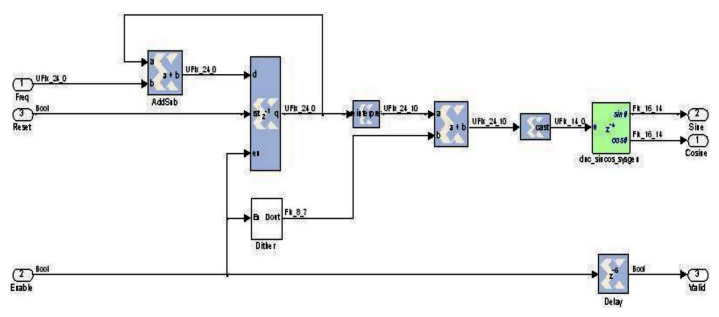

Fig. 3. Direct digital synthesizer.

Figure 3 shows the simulation block diagram of the direct digital synthesizer.

Figure 4 shows the half band filter, which has been used here to reduce the complexity of the multistage system. Figure 5 shows the output spectrum of three stage interpolator without half band filters.

Figure 6 shows the output spectrum of three stage interpolator.

The single stage interpolator produces stop band edge of $14.6 \mathrm{MHz}$, but for three stage interpolator this stop band edge reduces to $6.7 \mathrm{MHz}$ with sharper responses

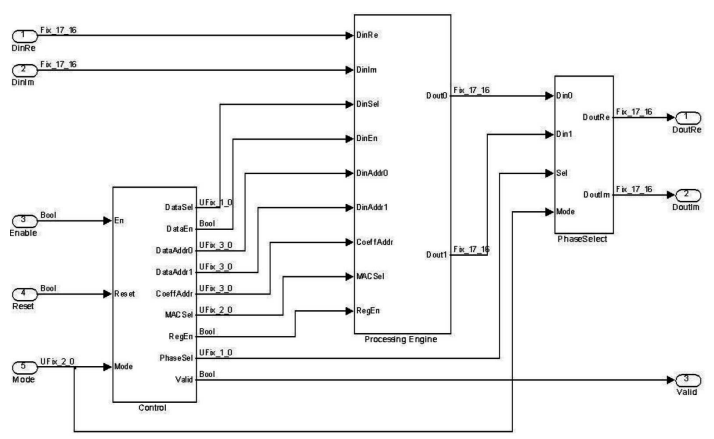

Fig. 4. Half band filter.

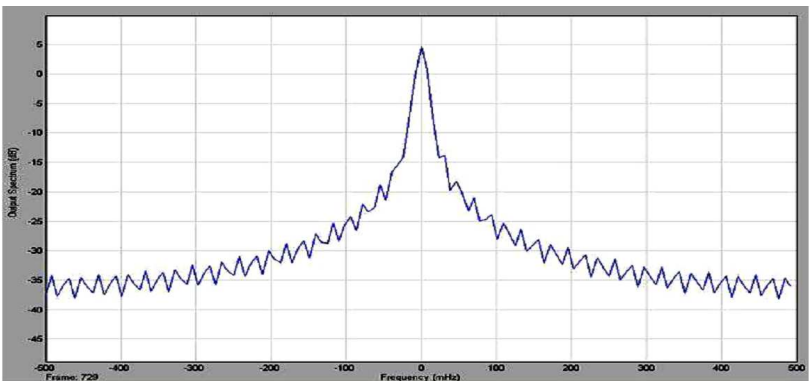

Fig. 5. Response of three stage interpolator without half band filters.

in stop-band region. Thus 3 -stage interpolation gives a sharper transition. The above results show that output spectrum of three stage interpolator is far better than the output spectrum of single stage. The pass band and stop band transition is much sharper and better in three stage interpolator.

\section{Conclusion}

As it is clear from the results using multistage interpolators, we can improve the efficiency of the system to a large extent which is the main requisite of digital communication systems nowadays. We can further improve the efficiency by cascading more and more interpolators, but the system becomes more and more complex as the number of stages increases. This complexity can be reduced drastically by using half band filters.

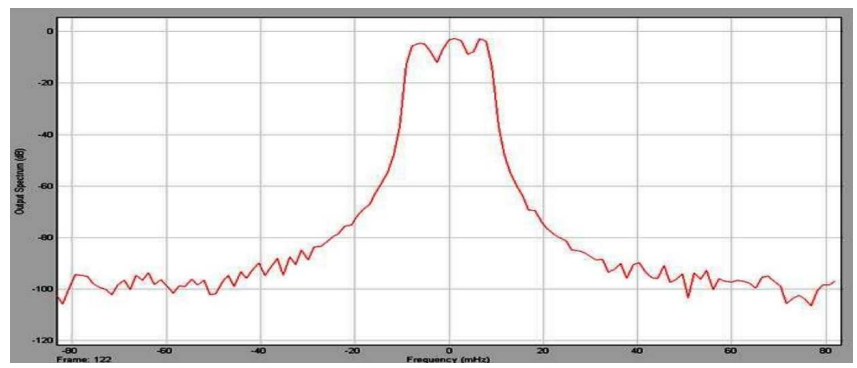

Fig. 6. Response of 3 stage interpolator using half band filter. 


\section{References}

[1] T. Saramaki, Handbook for Digital Signal Processing, Eds. S.K. Mitra, J.F. Kaiser, Wiley, New York 1993, p. 155.

[2] P.P. Vaidyanathan, T.Q. Nguyen, IEEE Trans. Circuits Syst. 34, 297 (1987)

[3] S.L. Freeny, J.F. Kaiser, H.S. McDonald, in: $A p$ plications of Digital Signal Processing, Ed. A.V. Oppenheim, Prentice Hall, Englewood Cliffs, NJ 1978, Ch. 1, p. 1.

[4] D.J. Goodman, J.L. Flanagan, in: Proc. IEEE Int. Communications Conf., Montreal, P.Q. Canada, Montreal 1971.
[5] R.G. Fridhamand, R.A. Mucci, Proc. IEEE 67, 904 (1979)

[6] L.R. Rabiner, B. Gold, Theory and Application of Digital Signal Processing, Prentice Hall, Englewood Cliffs, NJ 1975.

[7] C.E. Shannon, Proc. IRE 37, 10 (1949).

[8] R.E. Crochiere, S.A. Webber, J.L. Flanagan, Bell Syst. Tech. J. 55, 1069 (1976). 